\title{
HEMOPTISIS EN LA TUBERCULOSIS INFANTIL
}

\author{
Por los Dres, NORMAN MOLL. RAUL MATTE L, y SERGIO IBAÑEZ \\ Hospital L. Calvo Mackenna, Clinica Fediátrica dej Prof. A. Ariztía.
}

En ol estudio estadístico basado en ciones discrásicas sanguíneas. Así, Essen241 nifios hospitalizados en el Servicio feld', en 1932, estudió las alteraciones de Broncopulmonares durante los años hemodistróficas an 20 pacientes con he1945 y 1946, hemos encontrado una fre- moptisis, concluyendo que en la mayor cuencia de $5,3 \%$ de enfermos con he- parte no existen alteraciones; para este moptisis.

Cuadro $\mathbf{N}^{\circ} 1$

Porcentaje de hemoptisis. 1945-1946.

\begin{tabular}{lcccc}
\hline Tipo de The. & Ingresos & Hemoptoicos & Fallecidas & $\begin{array}{c}\text { Hemoptoicos } \\
\text { fallecidos }\end{array}$ \\
\hline Primaria & 196 & 6 & 21 & 3 \\
Post-primaria & 31 & 4 & 7 & 2 \\
Reinfecction & 14 & 3 & 5 & 1 \\
Totales & 241 & 13 & 36 & 6
\end{tabular}

Sólo: el $3 \%$ de las tuberculosis primarias presentan homoptisis. En cambio, en las formas post-primarias y en las de reinfección el porcentaje es mayor. En esta última categoría las lesiones son con mayor frecuencia del tipo cavilatio, favoreciendo, por lo tanlo, la aparicióu de las heuroptisis.

Patogenia de las hemoptisis.

En resumen, se considerau dos grupos die factores:

Factores primarios:

1) Alteraciones disctásicas de la sangro. lar.

2) Alteraciones del endotelio vascu-

3) Lesiones destructivas de las paredes de los vasos.

La mayoría de las investigaciones determinan que casi nunca existen altera- autor el factor fundamental en la hemoptisis es la lesión anatómica vascular.

En 12 enfermos que estudiamos de cerca, se practicó el tiempo de sangría y de coagulación. En todos el tiempo do sarigría fué normal. Todos, menos uno, tuvieron tiempo de coagulación normal.

Al lado de los factores primarios, influyen diversos factores secundarios:

1) Edad.

2) Labilidad neurovegetativa.

3) Vatiaciones meteorológicas. fera.

4) Grado de humedad en la atmós-

Consecuencias de las hemoptisis tubercnlosas.

Una hemoptisis puede provocar la muerte inmediata, como sucede en el caso de las hemoptisis fulminantes: o también puede provocar la muerte, determinando primero un cuadro de anemia aguda. Pero cuando no conduce a la 
muerte, puede suceder que en los díds que continúan a la bemoptisis se presente un cuadro febril, con aparición de signos estetoacústicos fugaces en la zona de la lesión sangrante. Este proceso se debe a una bronquioalveolitis secundaria, en cuyo tratamiento hemos obtenido muy buenos resultados con la administración de penicilina.

Pero es sobre todo interesante una complicación frecuente de las hemioptisis, que es la diseminación post-hemoptoica. Debemos distinguir: 1) Diseminación propiamente sanguínea, y 2) Diseminación tuberculosa.

En muchos casos, cuando la hemoptisis es abundante, al hacer un examen radiológico, nos encontramos con que tanto el lado sangrante como el opuesto, aparecen sembrados de una cantidad de sombras nodulares de tamaño variable. Se ba discutido mucho si la sangre puedo o no ocasionar imágenes semejantes, sin que exista por el momento acuerdo. Es evidente que cuando el volumen sanguíneo es suficientemente grande, da lugar a una sombra, pero es pteciso que el vaso sea de gran calibre, como por ejemplo, una rama principal de la arteria pulmonar.

Después de la hemoptisis aparecen sombras que por su tamaño deberán corresponder al grosor de uno de esos vasos finos y, sin embargo. tales nodulitos dan sombras intensas. Ello hace pensar que no representan solamente acúmulos de sangre.

Tampoco se puede pensar que sean lesiones específicas:

a) Porque aparecen inmediatamente cespués de la hemoptisis.

b) Porque en casi todos los casos nos encontramos con que transcurridas unas cuantas semanas, desaparecen.

Al aparecer una hemoptisis, en ol momento en que se pone en contacto con la mucosa de un bronquio la sangre, surge el reflejo de la tos y entonces es proyectada en forma diferente, según su cantidad y sus condiciones físico-químicas. $\bar{A}$ veces le tos dispersará cantidades peque:ñas de sangre que todavía no se ha coa- gulaćo. En otras veces, el árbol bronquial aparece inundado por una cantidad mayor en parte líquida y en parte coagulada.

He aquí las eventualidades posibles:

1) Aparte de la sangre vertida al exterior y de alguna dispersada en el parénquima, un coágnlo va a ocluir ul. bronquio principal, lobar o lobular. Se produce la atelectasia obstructiva posthemoptoica. (Obs, 225/42).

2) En otros casos el coágulo va a localizarse en un bronquio más pequeño o en el tronquiolo de un acini pulmonar. Teóricamente no debe originarse atelectasia, porque los poros de Kohn ponen en comunicación unos con otrós los alvéolos pulmonares, pero en el caso de la hemorragia la situación se aparta de la normalidad, determinando que las sombras más pequeñas en parte serán ocasionadas por la irritación de la sangre (bronco-alveolitis aguda) $y$ en parte serán también atelectásicas.

\section{Diseminación hemoptoica tuberculosa.}

Cuando predomina el componente lesional caseoso, la sangre se mezcla con la expcctoración bacilífera y constituye un vehiculo de difusión de los gérmenes en el parénquima pulmonar. El cuadro inmediato no se diferencia en nada del anterior, mas, al transcurrir los días, en vez de ceder los sintomas generales y reabsorberse los focos, nos encontramos con que persiste la fiebre, aumentan la tos y expectoración y observamos en la radiografía que mientras algunas sombras de diseminación comienzan a borrarse, otras se acentúan y aumentun de tamaño.

En el Servicio de Broncopulmonares hemos observado cuarenta enfermitos con hemoptisis. A esta casuística debemos agregar algunos enfermos del Sanatorio de Niños Josefina Martínez de Ferrari. En este último hay un buen núrilero de observaciones sobre el mismo tema, pero no se incluyen debido a ser adolescentes, de entre 15 y 18 años. 


\section{Cuadro No 2.}

Distribución por diagnóstico y presencia de cavernas.

\begin{tabular}{lcccc}
\hline Dlagnóstico & N* & Con cavernas & $\begin{array}{c}\text { Mort. e. } \\
\text { formas } \\
\text { cavitarias }\end{array}$ & $\begin{array}{c}\text { Mort. no } \\
\text { cavitarios }\end{array}$ \\
\hline Tuberculosis primaria & 6 & 3 & 1 & 0 \\
Tuberculosis post_primaria & 11 & 7 & 3 & 0 \\
Tuberculosis reinfeoción & 23 & 14 & 7 & 1 \\
\cline { 2 - 5 } & 40 & 24 & 11 & 1
\end{tabular}

El análisis del cuadro 2 señala que 6 de enfermos que presentaron diseminaenfermos tuvieron lesiones primarias; ción post-hemoptoica, es casi igual entre 11 post-primarias y el resto formas de los cavitarios que entre los no-cavitareinfección. Del total de casos, la mayo- rios.

ría de los fallecidos corresponden a las De los enfermos que han presentado formas cavitarias. Respecto al número diseminación, sólo 2 han fallecido.

Cuadro $\mathrm{N}^{4} 3$

Diseminación post-hemoptoica.

Tipo tuberculosis

Duración de la

Agravamiento

diseminación

S. C.

3 meses

Pasajero

Reinfección

A. D.

1 mes

Pasajero

Reinfección Caverna infjutrada

E. C.

4 meses

Pasajero

Reinfección. Swb-clavlcular

s. $\mathbf{P}$.

4 meses

Pasajero

Adenopatía Primaria

I. $\boldsymbol{R}$.

Adenopatía Primarla

R. A.

Rutinfección Caverna

4 meses Pasajero

M. A.

Reinfección

A. $T$.

Reinfectón

M. A.

Reinfección

7 meses (probable más) Fallecló (2 años después)

2 meses Pasajero

A. $\mathbf{S}$.

Reinfección

5 meses

Pasajero

3 meses

Pasajero

Atelectasla post-hemoptoica Pasajero (fallecló 2 años despues) 
Las diseminaciones post-hemoptoicas han ocurrido tanto en las formas cavitarias como en las no cavitarias. Diez niños presentaton diseminación post-bemoptoica.

Todos los casos, menos 1 (R. A.) parecen corresponder a la diseminación post-hemoptoica propiamente sanguínea, es decir, que las sombras de diseminación se deban a la repartición de la sangre en el árbol bronquioalveolar vecino. En cambio, en un caso estas lesiones persisten un mayor tiempo, provocando un agravamiento progresivo que al cabo de muchos meses lleva a la muerte.

La mitad de las hemoptisis ocurren en niños de más de 10 años: generalmente en casos de taberculosis de reinfección. En cambio, en las formas post-primarias $y$ en las formas primarias hay un me nor porcentaje. El niño de menor edad tenia 3 años.

Hay autores que señalan que las hemoptisis se presentan especialmente en ciertas localizaciones; otros suponen que son más frecuentes en las formas cirróticas y granúlicas. Nuestra apreciación es que en el niño parece ser más frecuente en las formas cavitarias.

De los 40 enfermos, 24 han tenido procesos bilaterales, correspondiendo en 6 casos a Granulia. Todas las formas granúlicas fallecieron. En Ios 16 enfermos restantes, en 9 había lesiones en el lado izquierdo, presentando sólo 2 un proceso de la base izquierda.

Las hemoptisis serían más frecuentes en las formas cirróticas y granúlicas. Nuestra apreciación es la de que en el niño hay un mayor porcentaje de formas cavitarias. La quinta parte de estos cavitarios presentan cavernas de localización parahiliar. Dada la cercanía de éstas a la zona hiliar, donde hay una gran riqueza vascular, se comprende la mayor facilidad para que se determinen hemoptisis. Tres enfermos presentaron, además de su praceso tuberculoso, una bronquiectasia y 1 caso la tisis coexistió con quiste hidatídico.

\section{Cuadro No 4}

Tratamiento colapsante.

\begin{tabular}{|c|c|c|}
\hline $\mathbf{N}^{2}$ & Lacalización & Tratamiento \\
\hline $10282-41$ & Bilateral. Ambas bases & Frenoparálisis \\
\hline $1447-43$ & Lobbulo metio derecho & Frenoparálisis \\
\hline $492-43$ & Infittractón base $\mathrm{y}$ vértice izquierdo & Frenoparálisis \\
\hline $4670-45$ & Subclavicular izquierda. Quiste hidatidico & Neumotórax \\
\hline $484-45$ & Subclavicular izqulenda. & Neumotórax \\
\hline $913-45$ & Caverna parahiliar izquierda & Neumotórax \\
\hline $1251-45$ & Caverna parahillar izquterda & Neumotórax \\
\hline $486-46$ & Lesión bilateral & Neumoperitoneo \\
\hline $600-46$ & Caterna parahiliar derecha & : Jacobaeus \\
\hline $4332-47$ & Adenopatia parahiliar izquierda & Frenoparálísls \\
\hline $\begin{array}{r}6398-47 \\
176-42\end{array}$ & $\begin{array}{l}\text { Lestón bilateral, Caverna superior izquierda } \\
\text { Tisis primaria derecha }\end{array}$ & $\begin{array}{l}\text { Frenop. Neumot. Neumoperit. } \\
\text { Neumotórax. }\end{array}$ \\
\hline $114-47$ & Caverna base derecha & $\begin{array}{l}\text { Neumot. Frenop. Neumope- } \\
\text { ritoneo relativamente eflcaz }\end{array}$ \\
\hline $2761-47$ & Infiltrado subclavicular derecho & Neumotórax \\
\hline $\begin{array}{l}2041-45 \\
4240-47\end{array}$ & $\begin{array}{l}\text { Granulfa. Proceso productivo base derecha } \\
\text { BilateraI }\end{array}$ & $\begin{array}{l}\text { Neumoperitoneo } \\
\text { Frenoparálisis }\end{array}$ \\
\hline
\end{tabular}


El estudio de la preferencia estacional de las hemoptisis y de su calidad, permite apreciar que, en la mayoría de los enfermos, presentan preferencia por las estaciones extremas (verano e invierno).

Respecto al tipo de las hemoptisis, goneralmente aparecen en un mismo enfermo todos los tipos. En 6. enfermos hubo hemoptisis de tipo fulminante, es decir, mortal de inmediato.

El mayor número de los fallecidos estuvo entre los que presentaron una sola hemoptisis.

Las hemoptisis de cierta consideración, especialmente en adolescentes y niños mayores, suelen producir en los dias siguientes a su producción un cuadro climico bien característico con disnea, fiebre y auscultándose a veces gran riqueza de ruidos broncoalveolares en el lado en que haya ocurrido la hemoptisis. Resultados muy ofectivos se obtienen en estos cuadros de bronquio-alveolitis post-hemoptoica con la administración de penicilina a las dosis usuales en las neumopatías agudas.

El estudio anátomo-patológico de los enfermos fallecidos permite señalar algunas lesiones principales:

\begin{tabular}{lc}
\hline Tipo de la lesión & Ni de casos \\
\hline Granulia & 6 \\
Caseosis & 9 \\
Bronquíectasla & 1 \\
Diseminación noduiar-caseosa & 1 \\
Cavernas & 8 \\
Roturas vasculares & 3 \\
Hemorragias & 3 \\
El examen radiológico de las lesiones \\
de los fallecidos permite señalar algunas \\
características generales, como ser: le- \\
sión bilateral, postprimaria o de reinfec- \\
ción, casi siempre cavitarias.
\end{tabular}

\section{Tratamiento de la hemoptisis.}

No hay uniformidad en cuanto a las medidas tendientes a cohibir una hemoptisis y a evitar su repetición.
Así, por ejemplo, Loeper ${ }^{5}$ recomienda las siguientes medidas:

1. Reposo.

2. Morfina.

3. Inyección de extracto de lóbulo posterior de hipófisis.

4. Inyecciones de cloruro de calcio.

5. Aplicación de un neumotórax de urgencia.

Giraud ${ }^{4}$ insiste en que la terapéutica medicamentosa es inútil, salvo en lo que se refiere al lóbulo posterior de la bipófisis. La acción hemostática del neumotórax presentaría resultados a menudo notables, a tal punto que Dumarest lo asemeja a la acción de una ligadura. Considera a la frenoparálisis tan útil como el neumotórax.

En las hemoptisis graves, Valdivieso señala dos recursos que habitualmen. te consiguen cohibir la hemorragia: las transfusiones y la colapsoterapia.

En el Servicio de Broncopulmonares del Hospital Calvo Mackenna se utiliza el siguiente esquema de tratamiento de la hemoptisis:

1. Reposo absoluto.

2. Morfina.

3. Penicilina (cuando a la hemoptisis sigue una bronquio alveolitis post-hemoptoica).

4. Colapsoterapia, cuyo tipo y oportunidad dependen más del tipo de la lesión que de la calidad y cantidad de la hemoptisis.

5. Transfusiones, indicadas en las hemoptisis abundants.

\section{Resultado del tratamiento en 40 hemoptoicos.}

Excelente resultado se ha obtenido en nuestros enfermos tratados mediante la colapsoterapia; especialmente mediante el neumotórax y la frenoparálisis. No así con el uso del neumoperitoneo; pero debemos tomar en cuenta que el neumoperitoneo ha sido aplicado en aquellas oportunidades en que todos los demás tratamientos habían ya fracasado. 
Resultado del tratamiento

$N^{*} \quad$ Fracasos Muertes

$\begin{array}{llll}\text { Neumotórax } & 6 & 2 & \\ \text { Frenoparálisis } & 6 & & 1 \\ \text { Neumoperitoneo } & 4 & 3 & 4 \\ \text { Jacobaeus } & 1 & & \\ \begin{array}{l}\text { Tratamiento no co- } \\ \quad \text { lapsante }\end{array} & 21 & 4 & 7\end{array}$

Respecto a las transfusiones, en aquellos casos en que se han empleado, lo han sido con la indicación de corregir una auemia, sea ésta del tipo de la anemia aguda, o bien, de instalación paulatina.

\section{Resamen.}

Se revisan 40 enfermos hospitalizados en el Servicio de Broncopulmonares del Hospital Calvo Mackenna, que presentaron hemoptisis durante el curso de su hospitalización. Doce enfermos fallecen.

Se revisan las complicaciones que aparecen en el curso de la enfernedad a consecuencia de la hemoptisis; algunos enfermos fallecen durante la hemoptisis; un porcentaje grande $(25 \%)$ presenta la aparición clínica y radiológica de una diseminación post-hemoptoica, que la mayoría de las veces es de carácter sanguíneo y no tuberculoso.

Se llega a la conclusión que el tratamiento más útil de la bemoptisis estriba en la colapsoterapia, preferentemente en el neumotórax y en la frenoparálisis. Las indicaciones de cada una de estas intervenciones está supeditada más al tipo de la lesión que a la calidad de la hemoptisis. En los enfermos tratados con medios no colapsantes, el número de fracasos es bien elevado (61 \%).

\section{Summary.}

40 patients with hemoptisis were considered from the point of view of etiology, mortality and treatment, 12 died. All had lung Tbc.

The complications appeared during the onset of the disease due to the hemoptisis are analised; some died during the hemoptisis: a higher incidente of cases showed the radiological and clinical installment of a post-hemoptoic disemination. most of the cases due to the blood sprayed through the bronchia.

The more usefull treatment of hemoptisis is collapse, neumothorax or frenoparalisis. The indications of either of these methods belongs more to the kind of the lung lesions than to the quality of hemoptisis. Among the patients whose hemoptisis were treated with no collapsant means the percentage of failure is too high.

\section{Bibliografía.}

1.-TAPIA. MANUEL. -- "Formás Anátomo-clinicas, diagnóstico y tratamiento de la tubercalosis pu'monar". Librería Luso-Española. Lisboa, 1945.

2.-HERNANDEZ DIAZ, A. - "Estudio sobre las hemoprisis neurovegetativas". Schweiz, Med. Wachr. 8: 1940.

3.-VALDIVIESO, RAMON. - "Tratado de Terapéutica Clínica". 1948. Central de Fublicaciones. Santiago.

4.-GIRAUD. - "Précis de Phtisiologie". París. 1939.

5.-LOHFER. - "Therapentique Medicale. Coent et Sang". Masson et Cie. Paris. 1933.

6.-DE RUDDER. - "Kinderartzliche Notfaltfibell". Georg Thisme Verlag. Stūttgatt, 1949. 